\title{
Study of effect of the inductor on dynamical behavior for electronic circuit by using nonlinear part from type memductance
}

\author{
Abo-Talib .Y. Abbas \\ Ministry of Education / General Directorate of Education in Basra / Qamar Bani Hashem Intermediate School, \\ *Corresponding Author: ttjeo76@gmail.com.
}

Received 11-02-2021, Accepted 16-03-2021, published 14-04-2021.

DOI: 10.52113/2/08.01.2021/98-108

\begin{abstract}
The effect of the coil on the dynamical behavior of the electronic circuit with nonlinear part memductance was studied, where we studied four coil values: $\mathrm{L}=90 \mathrm{mH}, \mathrm{L}=80 \mathrm{mH}, \mathrm{L}=70 \mathrm{mH}$ and $\mathrm{L}=100 \mathrm{mH}$ with changing the value of the first capacitance $c_{1}$. We obtained stability maps consisting of two regions (the dynamical behavior zone and the stability zone), where the dividing line is the Hopf bifurcation line, as well as the bifurcation diagram for each value from the coil and the first capacitance was obtained, as well as the phase portrait diagram in which we obtained the chaotic attractions And the period of the first, second, third, fourth, fifth, sixth and eighth.
\end{abstract}

(C) 2021 Al Muthanna University. All rights reserved.

Keywords: chaos, Lorenz, Chua, Bifurcation point, equilibrium.

\section{Introduction}

The occurrence of the phenomenon of chaos in electronic circuits has great importance in terms of the wide field in its practical applications, for example, but not limited to, Through chaos technology, it is possible to hide many information that is not intended to be leaked to other parties [1], Likewise, through the technology of chaos in electronic circuits, it is possible to control information systems and control the mechanism to direct them $[2,3,4]$, They also use them to obtain desirable sounds in instruments musical [5]. Scientist Chua was the first to try to obtain on the chaos on electronic circuits in 1983 when he visited Matsumoto's lab [6,7]. At Waseda University, Chua's beginnings were different from those of Lorenz [8] and Rossler [9], as he studied the two systems and identified the points and conditions for chaos in these two systems, then he proceeded to apply these conditions to electronic circuits, Since the scientist Chua determined that he needs at least two points of equilibrium, in addition to a non-linear part, 
so the Chua scientist needs three energy storing elements in order to give a derivation to time and these energy storing elements either as coils (where the voltage is the change of current with time), Or capacitors (where the current is a time-varying voltage). Exclude the scientist Chua, that the circuit is made of coils only or capacitors only because it can be reduced to only one element. Therefore, he decided that it should be from coil s and capacitors, but he preferred that the circuit be of two capacitors and a coil instead of two coils and capacitor, because of the ease of obtaining the appropriate capacity as well as the cheap price, The scientist Chua preferred the parallel circuit to be similar to a parallel resonance circuit. . After Chua finished identifying the energy storing elements, he focused his efforts on the nonlinear part and how to choose it, because the scientist Chua identified three equilibrium points, so he preferred that the nonlinear part be from three regions, each region with a negative slope [10], So he suggested a nonlinear part of the piecewise linear, then the scientist Chua added a constant resistance, After doing computer simulations of the circuit, scientist Chua got the chaotic attraction he was looking for in electronic circuits. It is possible to distinguish between the phenomenon of chaotic and the phenomenon of random noise that occurs frequently in dynamical systems in two ways $[11,12]$ :

1. The power spectrum characteristic of the broadband system output signal by using the fast Fourier transform technology.

2. The exponential spacing characteristic of chaotic system paths for different initial conditions infinitely small, as the paths start very close to each other and then quickly diverge from each other exponentially with time, and this can be verified by the Lyapunov guide, where the greater the value of the Lyapunov index increases Spacing between paths that are close to each other in the beginning.

The search order will be as follows, the first section will review the non-linear circuit studied, followed by the second section dealing with the study of stability maps and the effect of the coil on them, while the third section will deal with bifurcation diagrams at different values of the first capacitor, and the fourth section will discuss phase portrait diagrams.

\section{The chaotic electronic circuit with a non- linear part is of the memductance type}


We will adopt the chaos circuit shown in Figure (1). Where R represents the linear resistance, $\mathrm{G}_{\mathrm{N}}$ represents the impedance, $c_{1}$ and $c_{2}$ the first and second capacitance, $\mathrm{L}$ the inductance of the used coil, $\varphi$ represents the magnetic flux, $q$ represents the electric charge, $v_{c 1}$ and $v_{c 2}$ the voltage around the two capacitors respectively, $i_{L}$ the current passing through the coil. For the purpose of studying the stability or instability of this circuit, the nonlinear part denoted by the symbol $\mathrm{M}$ has been added, which is of the type Memristor flux-controlled (memductance)[13-16].

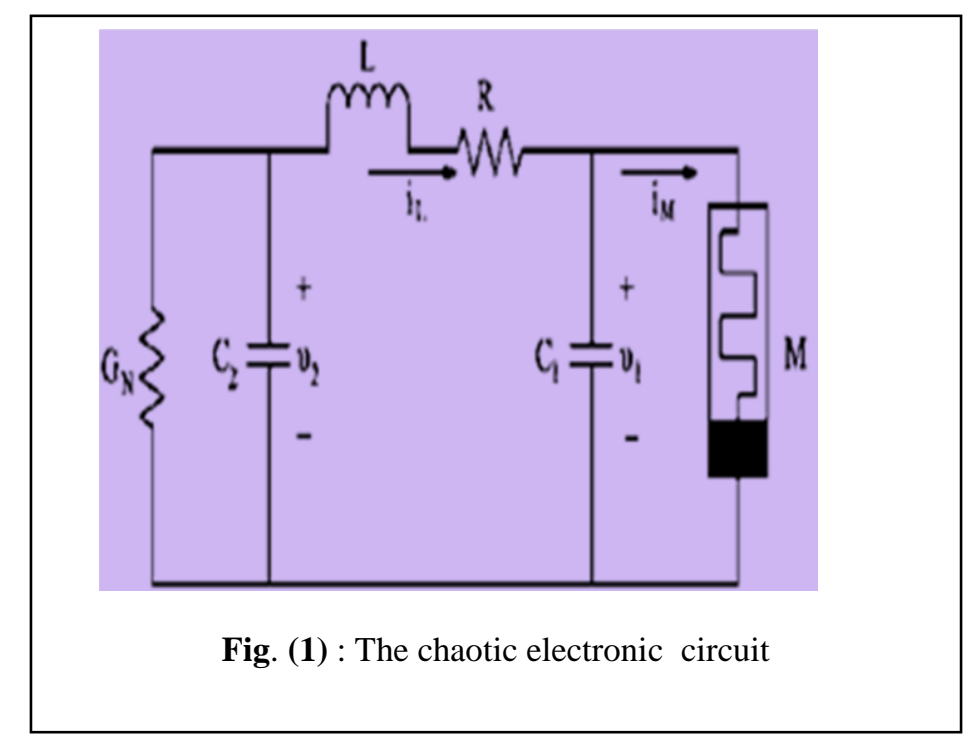

Now, by applying Kirchhoff's laws about the first and second amplitudes, the coil and the resistance, we will obtain the differential equations shown below:

$\frac{d \varphi}{d t}=v_{c 1}$

$\frac{d v_{c 1}}{d t}=\frac{1}{C_{1}}\left(i_{l}-w(\varphi) v_{c 1}\right)$

$\frac{d v_{c 2}}{d t}=-\frac{1}{C_{2}}\left(i_{l}+G_{N} v_{c 2}\right)$

$\frac{d i_{l}}{d t}=\frac{1}{L}\left(v_{c 2}-R i_{l}-v_{c 1}\right)$

Where $\mathrm{w}(\varphi)$ represents the function of the nonlinear part and that this portion follows the behavior of the equation below:

$$
w(\varphi)=\frac{d q(\varphi)}{d \varphi}=-\alpha+3 b \varphi^{2}
$$


Where $\alpha$ and $b$ are constants. The above equations were solved using the fourth-order Rang-kta method using the Fortran 90 programming language and the boundary conditions were, $\mathrm{i}_{2}(0)=0.0, \mathrm{i}_{1}$ $(0)=0.001, v_{c 1}(0)=0.006, v_{c 2}(0)=0.02, \mathrm{~h}=0.1 \mathrm{e}-5, \mathrm{~g}=0.4 \mathrm{e}-4, \mathrm{~b}=4.0 \mathrm{e} 4$, Alfa $=0.5 \mathrm{e}-4$.

\section{Stability maps}

Figure (2) represents the stability maps for the Memductance circuit, which are divided into two main areas: the stability zone with green color and the dynamical behavior area with yellow color. The stability zone means that all the dynamical behaviors of the electronic circuit under study are fixed with time and in the phase portrait these behaviors appear in the form of a fixed point. As for the dynamical behavior area, it includes all kinds of behaviors, including period doubling, quasi periodic and chaotic, which can be obtained as a result of changing some variables such as $\left(c_{1}\right.$, $\left.c_{2}, L, R\right)$. The use of stability maps is of great benefit as it gives a comprehensive understanding of the behavior of the system in a space in which two of the variables under study change and which cannot be observed in other methods used to describe the stability state of the system (such as a bifurcation diagram, phase portrait, Poincare section and other methods. ).

In Fig. (2) we note the stability maps in the plane $\left(c_{2}-c_{1}\right)$ for different values of self inductance of the coil $(\mathrm{L}=100 \mathrm{mH}, 90 \mathrm{mH}$, $80 \mathrm{mH}, 70 \mathrm{mH})$. Moving from the value of $\mathrm{L}=100 \mathrm{mH}$ to the value of $\mathrm{L}=90 \mathrm{mH}$ does not lead to an increase in the dynamical behavior area, but that the size of that area remains constant despite a change in the extent of its appearance and disappearance, so we find that it begins at $\mathrm{c}_{2}=53.0 \mathrm{nF}$ and ends at $\mathrm{c}_{2}=54.4$ $\mathrm{nF}$ in the case of $\mathrm{L}=100 \mathrm{mH}$, While it starts at $\mathrm{c}_{2}=46.2 \mathrm{nF}$ and ends at $\mathrm{c}_{2}=47.6 \mathrm{nF}$ for the resistance value $\mathrm{L}=90 \mathrm{mH}$. But we find that there is a decrease in the dynamical behavior area (although not very clear)) when moving to the state of $\mathrm{L}=80 \mathrm{mH}$, but this decrease in the dynamical behavior area is clearly visible in the case of $\mathrm{L}=70 \mathrm{mH}$, which we notice in this case is the disappearance of the area of dynamical behavior completely and then reappear, and this means that there is more than one area of dynamical behavior of the circuit separating those areas a zone of stability. The boundary between the stability zone and the dynamical behavior zone (yellow) represents the values of the Hopf bifurcation, at which the dynamical behavior begins, transforming from the onecycle behavior of P1 to the fixed point. The dynamical behaviors of the yellow area are shown in the bifurcation figures (3), (4) and (5).

Knowing that the values of the two amplitudes, the coil and the resistance are practical values, 
meaning that the application of these values in

practice leads to the same theoretical results.
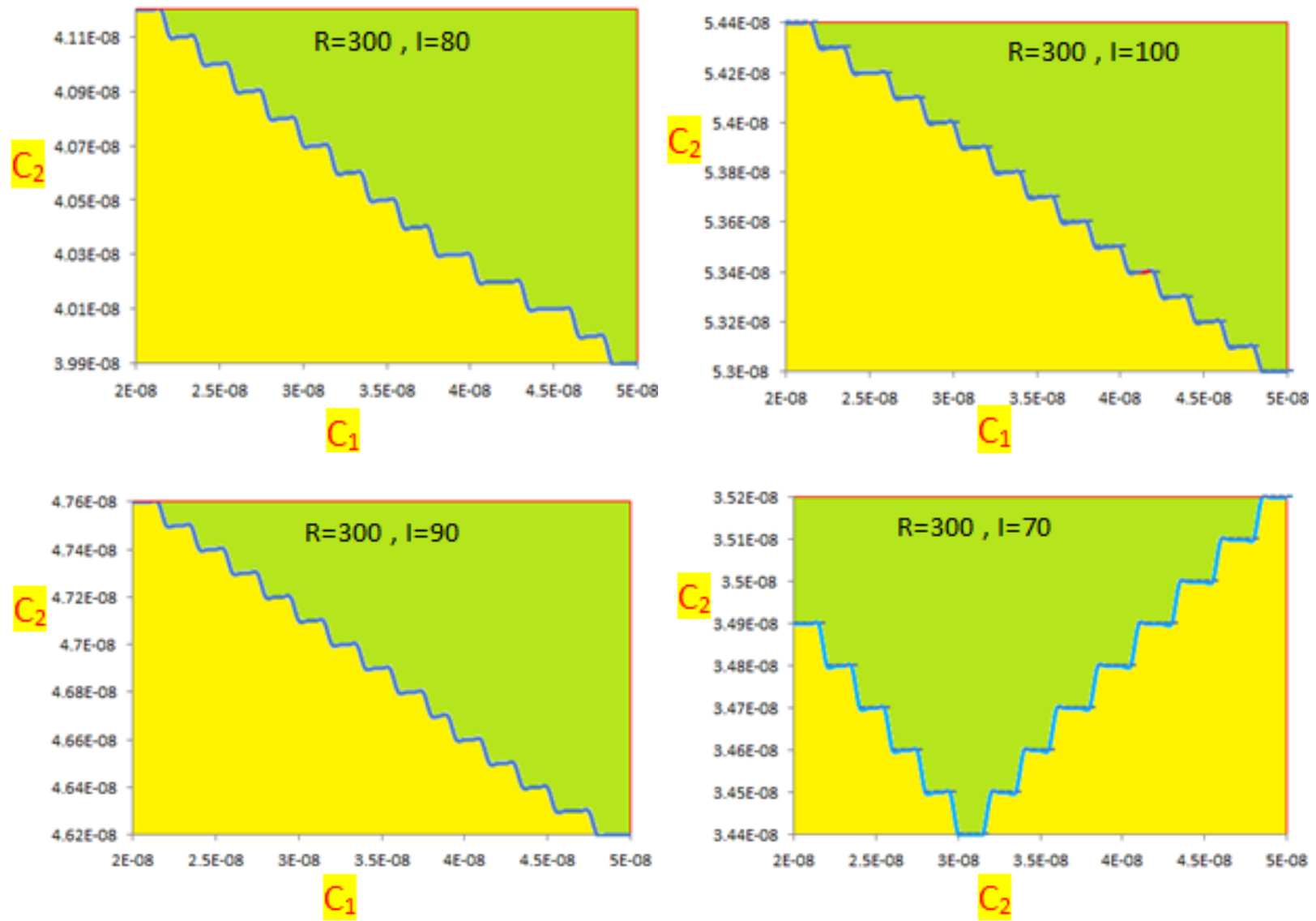

dynamical behavior region.

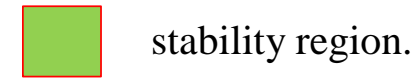

stability region.

Hopf bifurcation

Fig.(2) : stability Map in plan $\left(C_{2}-C_{1}\right)$ for change in value $L$.

\section{Study of the diagram bifurcation}

The description of the dynamical behavior of the electronic circuit represented by the stability maps shown in Fig. (2) was studied by drawing the bifurcation shapes shown in Figures (3), (4) and (5), in the plane $\left(\mathrm{V}_{\mathrm{c} 2}-\mathrm{c}_{2}\right)$ and for different values of $c_{1}$ within the studied range in the stability maps at the value of $\mathrm{L}=100 \mathrm{mH}, \mathrm{L}=90 \mathrm{mH}$ and $\mathrm{L}=80 \mathrm{mH}$. To get a more accurate description of these behaviors, we will work on the use of stability maps and bifurcation shapes together. From the figure(3), (4) and (5), with the help of the stability map (Figure 2), it can be seen that:

1- The presence of a diversity in the dynamical behavior, in terms of the emergence of various periodic behaviors with the presence of areas of chaos.

2- All the figures in figure (3) show that the behavior of this circuit and for the values of $c_{2} \geq 44.0 \mathrm{nF}$ shows the 
behavior of one period P1 until it reaches the value of Hopf bifurcation, which means the transformation of the circuit's behavior to the stable behavior that appears in the phase portrait as a fixed point. While, all figures in Figure (4) show that the behavior of this circuit and for the values $c_{2} \geq 39.0 \mathrm{nF}$ shows the behavior of one cycle P1 until it reaches the value of Hopf bifurcation. The same thing happens in Figure (5), where all the figures show that the behavior of this circuit and for values $\mathrm{c}_{2} \geq 33.0 \mathrm{nF}$ shows the behavior of one cycle P1 until it reaches the value of Hopf bifurcation.

3- At $\mathrm{L}=100 \mathrm{mH}$, for values of $\mathrm{c}_{1}>$ $35.0 \mathrm{nF}$, the behavior of the electronic circuit becomes periodic (i.e. the chaotic behavior disappears), as we observe $\mathrm{P} 1, \mathrm{P} 2, \mathrm{P} 4$ at the value of $\mathrm{c}_{1}=$ $36.0 \mathrm{nF}$, and then the cycle time behavior of one and two (P1 and P2) appears only at the value of $\mathrm{c}_{1}=38.0 \mathrm{nF}$. By increasing $c_{1}$, the behavior of the electronic circuit will be limited to that of one period P1. While, at $\mathrm{L}=90 \mathrm{mH}$, for values of $c_{1}>31.0 \mathrm{nF}$, the behavior of the electronic circuit becomes periodic (i.e. the chaotic behavior disappears), as we observe P1, P2, P4 at the value $\mathrm{c}_{1}=32.0 \mathrm{nF}$, and then the cycle time behavior of one and two (P1 and P2) appears only at the value of $c_{1}$ $=34.0 \mathrm{nF}$. By increasing $\mathrm{c}_{1}$, the behavior of the electronic circuit will be limited to that of one cycle P1. The same thing happens at $\mathrm{L}=80 \mathrm{mH}$, for values of $\mathrm{c}_{1}>$ $26.0 \mathrm{nF}$, the behavior of the electronic circuit becomes periodic (i.e. the chaotic behavior disappears), as we observe the behavior of P1, P2, P4 at the value $c_{1}=28.0 \mathrm{nF}$, and then the cycle time behavior of one and two (P1 and P2) appears only at the value of $c_{1}$ $=30.0 \mathrm{nF}$. By increasing $\mathrm{c}_{1}$, the behavior of the electronic circuit will be limited to that of one cycle P1.

4- In the figure (3), the chaotic regions within the range of $20.0 \mathrm{nF} \leq \mathrm{c}_{1}<35.0 \mathrm{nF}$ contain periodic behaviors (windows) that sometimes appear within a wide range of $c_{2}$ values as the doubling of period three and six (P3, P6) shown in the figure (3). While, in the figure (4), the regions of chaos within the range $20.0 \mathrm{nF} \leq \mathrm{c}_{1}<31.0 \mathrm{nF}$ contain within them periodic behaviors (windows) that sometimes appear within a wide range of $c_{2}$ as the doubling of cycle time three and six (P3, P6) shown in the figure (4). The same thing happens in the figure (5), The chaotic regions within the range $20.0 \mathrm{nF} \leq \mathrm{c}_{1}<26.0 \mathrm{nF}$ contain 
within them periodic behaviors doubling of cycle time three and six (windows) that sometimes appear (P3, P6) shown in the figure
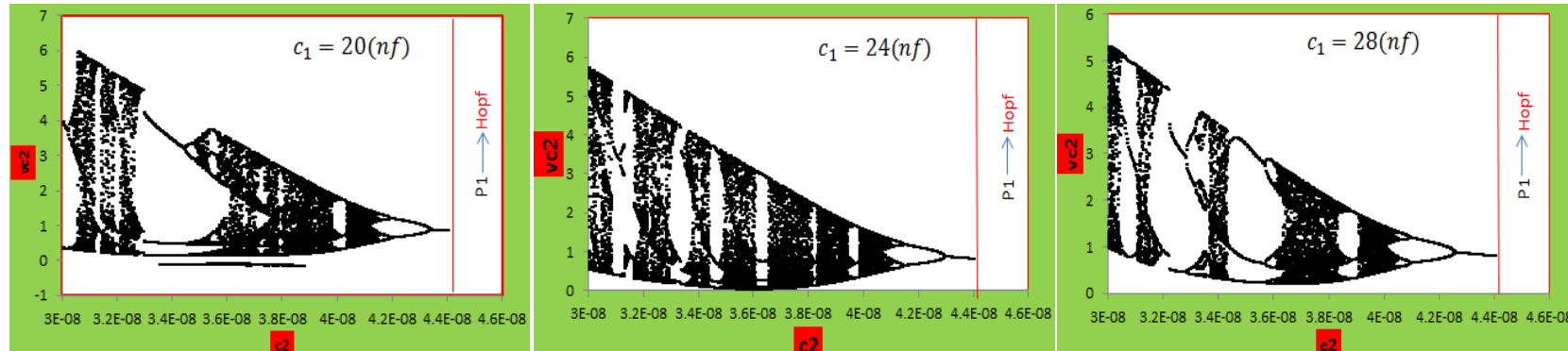

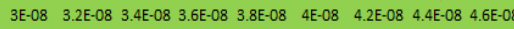
c2

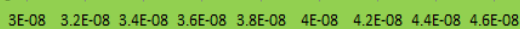
c2
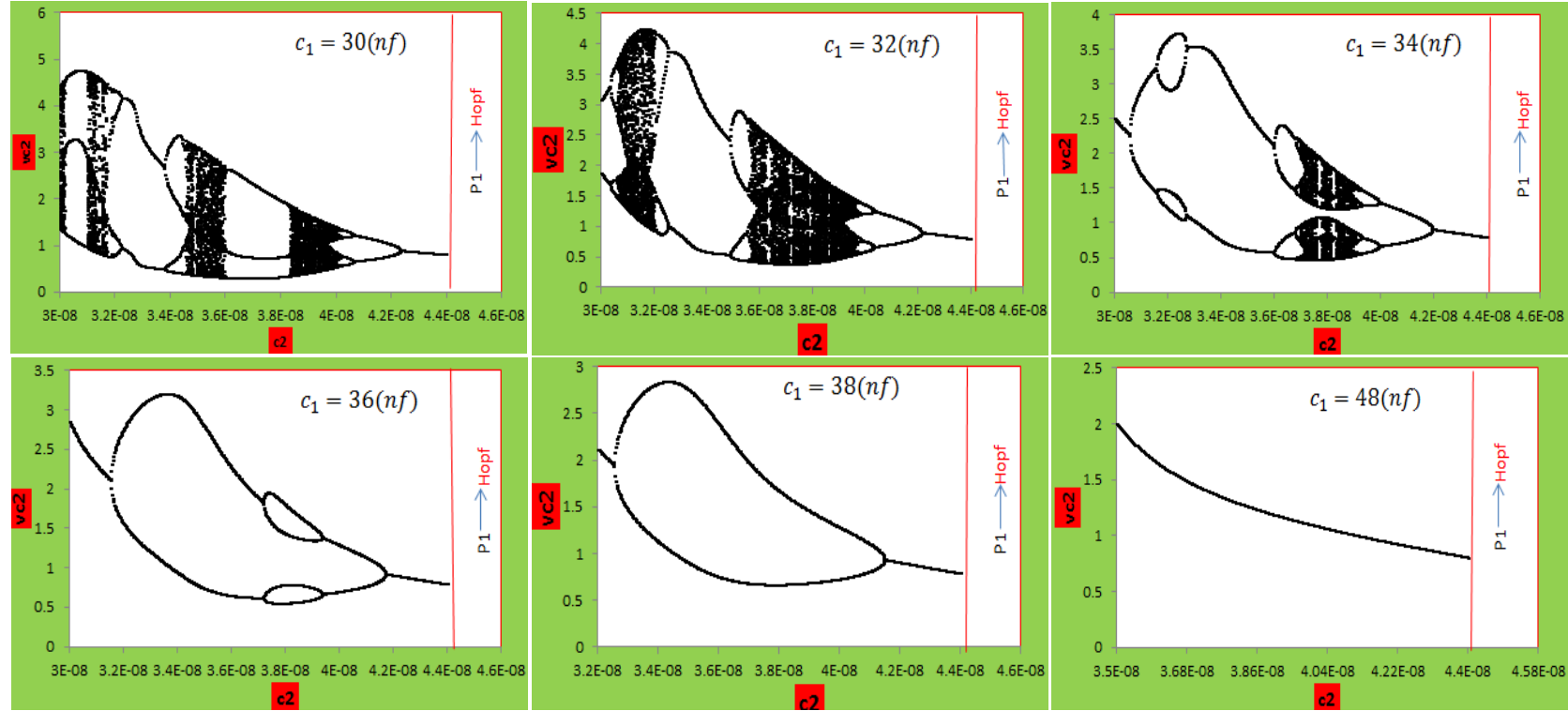

within a wide range of $c_{2}$ as the

Fig. (3) : scheme bifurcation for value $L=100 \mathrm{mH}, \mathrm{R}=300 \Omega$.
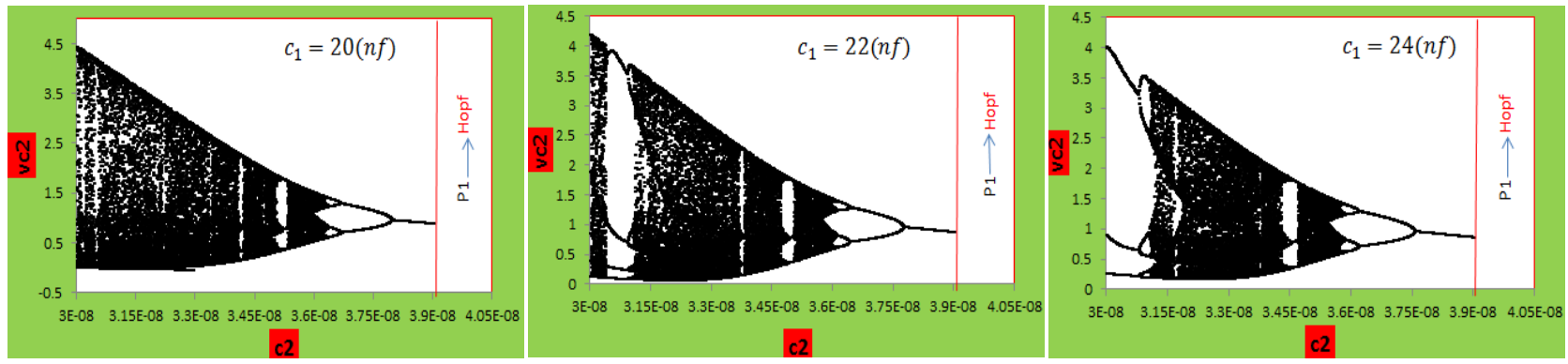

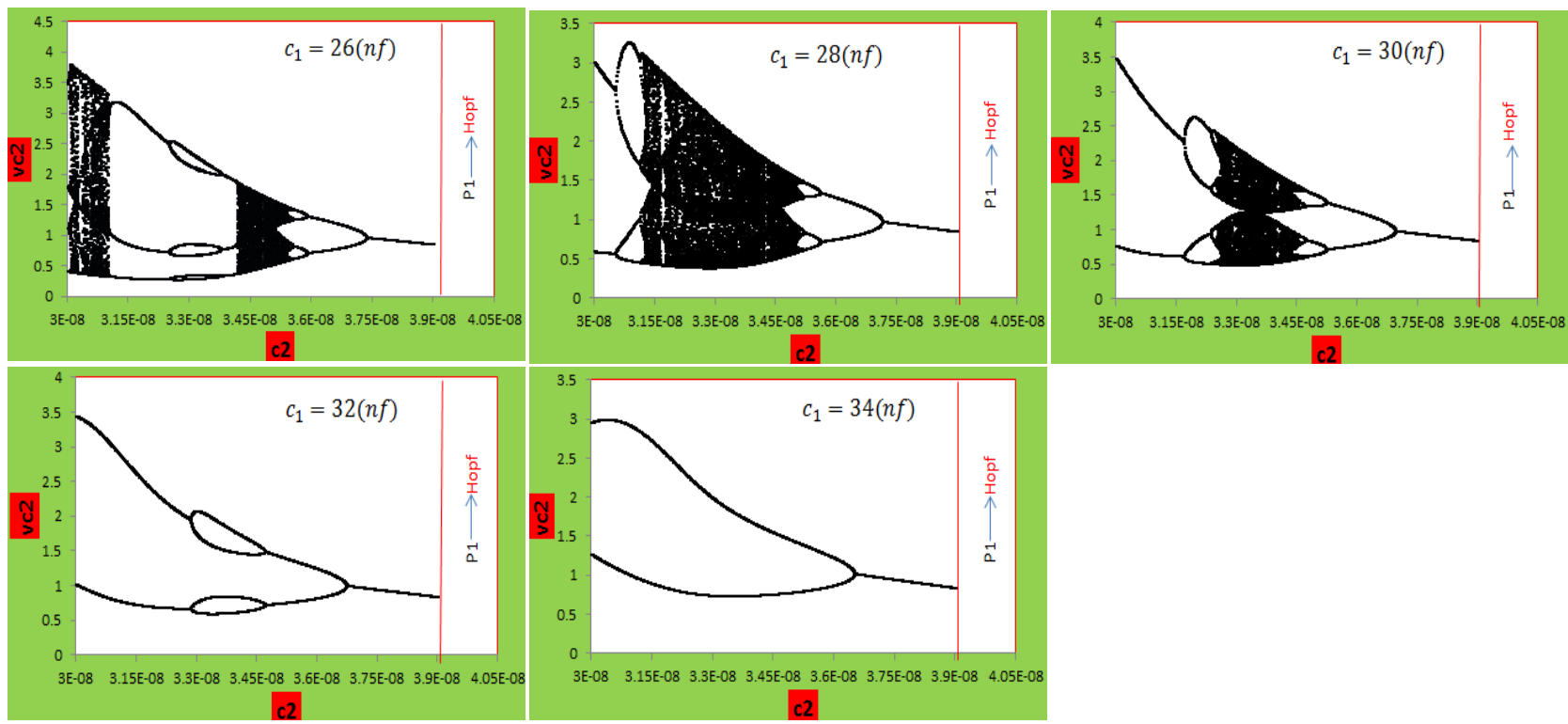

Fig.(4): scheme bifurcation for value $L=90 \mathrm{mH}, \mathrm{R}=300 \Omega$.
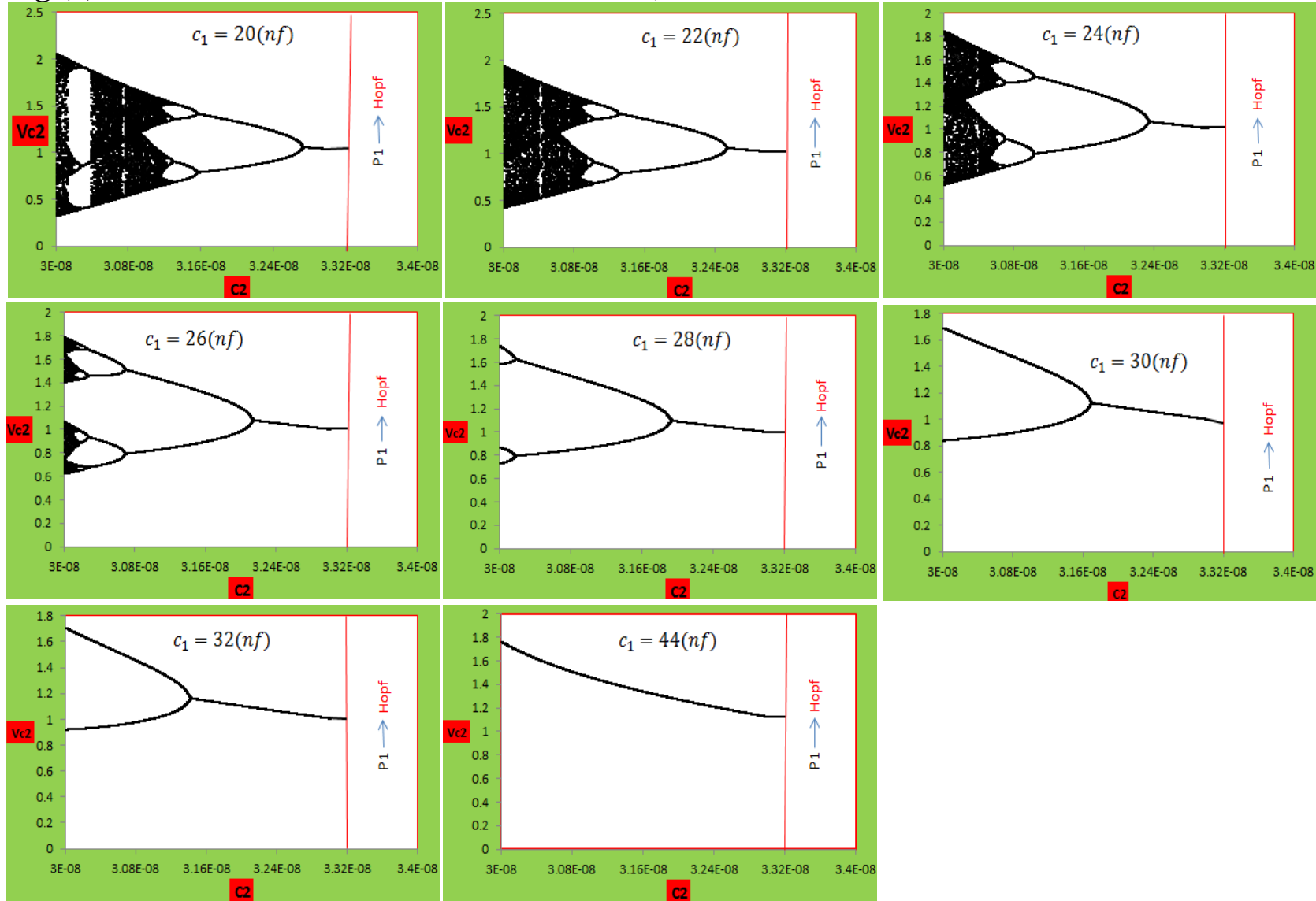

Fig. (5) : scheme bifurcation for value $L=80 \mathrm{mH}, \mathrm{R}=300 \Omega$. 
the bifurcation diagram shown in Fig. 3, Fig. 4 and Fig. 5 was studied by drawing the phase portrait shapes shown in Fig. 6 and Fig. 7 and Fig. 8 in the plane $\left(\mathrm{V}_{\mathrm{c} 1}-\mathrm{V}_{\mathrm{c} 2}\right)$ and different values of $c_{2}$ in order to obtain a more accurate description of these kinematic behaviors.
Due to the large number of bifurcation diagrams that we studied, we cannot study each diagram in the form of a phase portrait diagram. Therefore, we chose only one form of bifurcation diagrams and the phase portrait diagram was studied for it, and we have chosen:

1- Dynamical behavior at $c_{1}=20 ., \mathrm{R}=300, \mathrm{I}=80$, where we obtained a variation in dynamical behavior that included both(Fig.6): a- period one p1 b- period two p2 c- period four P4 d- attractor chaotic $\quad$ g- period three p3 h- period six p6.

2- Dynamical behavior at $\mathrm{c}_{1}=26$., $\mathrm{R}=300, \mathrm{I}=90$, where we obtained a variation in dynamical behavior that included both(Fig.7) : a- period four p4

b- period five $\mathrm{p} 5$ c- attractor chaotic d- period three p3 $\quad$ e- period six p6 $\quad$ f- period two P2 g- period one $\mathrm{P} 1$.

3- Dynamical behavior at $\mathrm{c}_{1}=28$. $\mathrm{R}=300, \mathrm{I}=100$, where we obtained a variation in dynamical behavior that included both(Fig. 8): a- attractor chaotic b- period three p3 c- period two p2 d- period eight p8 e- period four p4 f- attractor chaotic g- period seven $\mathrm{p} 7 \quad \mathrm{~h}$ - period one $\mathrm{p} 1$.
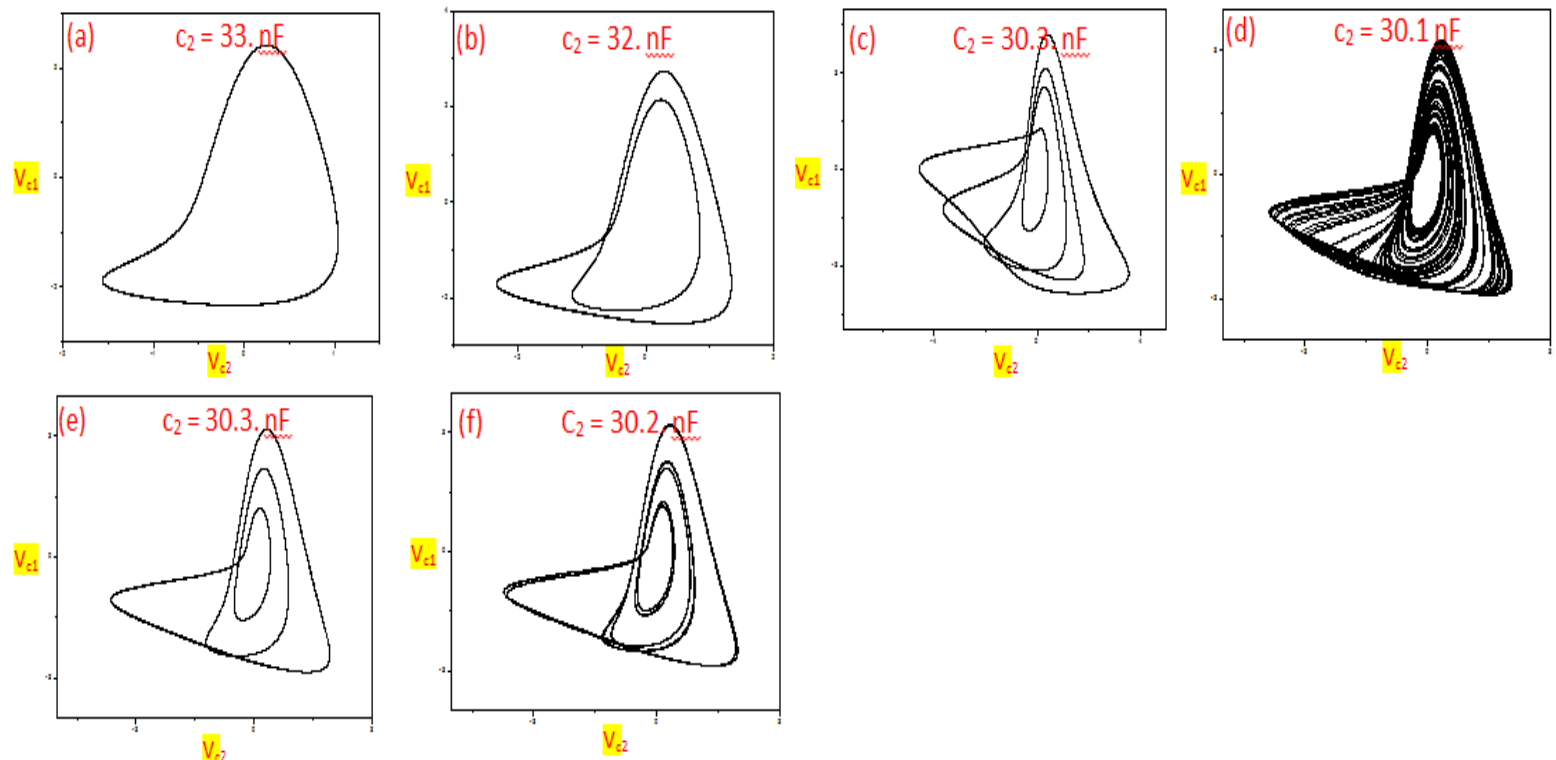

Fig. (6) : scheme phase portrait for value $\mathrm{L}=80 \mathrm{mH}, \mathrm{R}=300 \Omega, c_{1}=20$. 

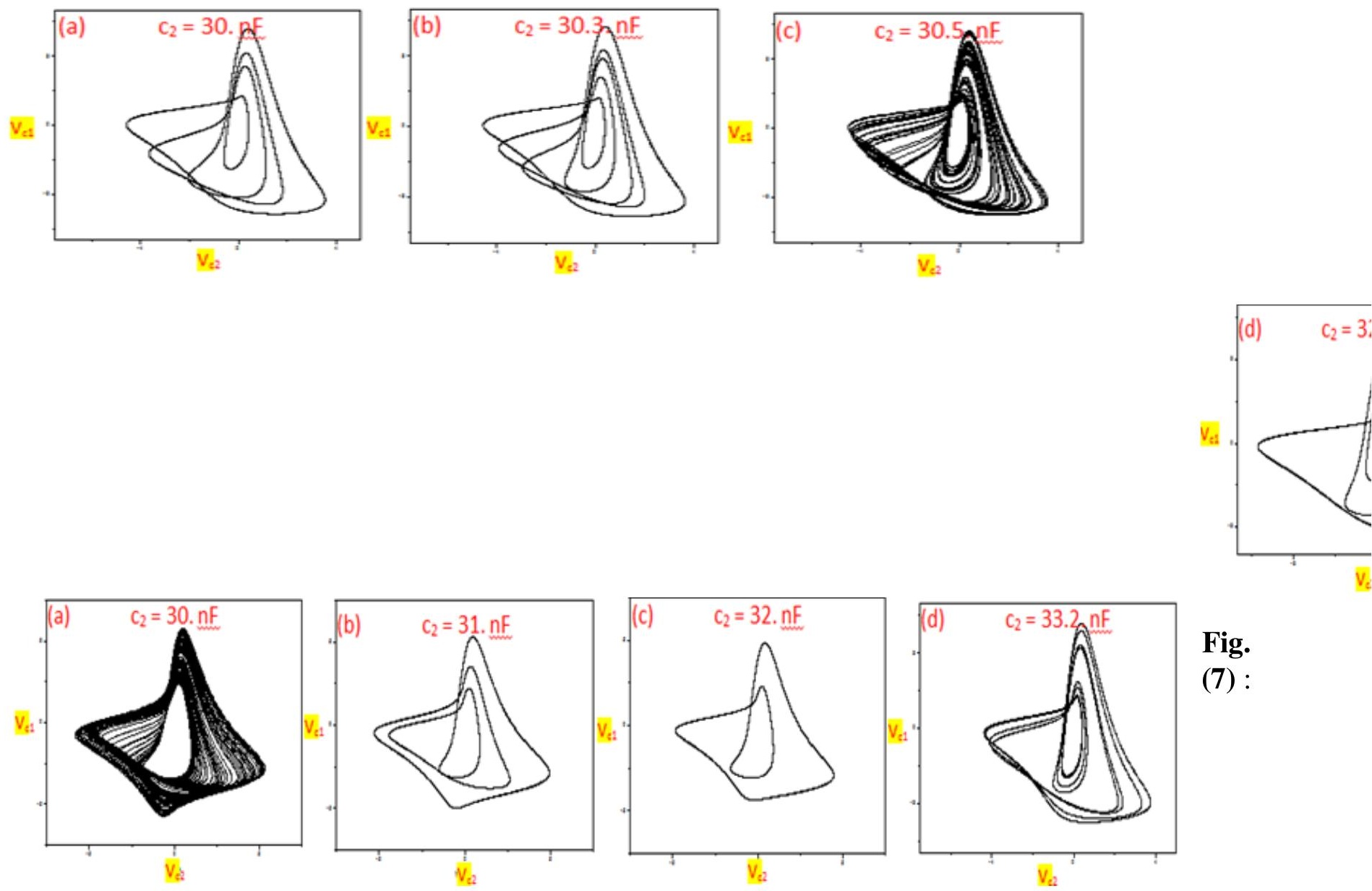

Fig.

(7) :

scheme phase portrait for value $\mathrm{L}=90 \mathrm{mH}, \mathrm{R}=300 \Omega, c_{1}=26$.

Fig.(8) : scheme phase portrait for value $\mathrm{L}=100 \mathrm{mH}, \mathrm{R}=300 \Omega, c_{1}=28$.

Conclusions: After we studied the stability
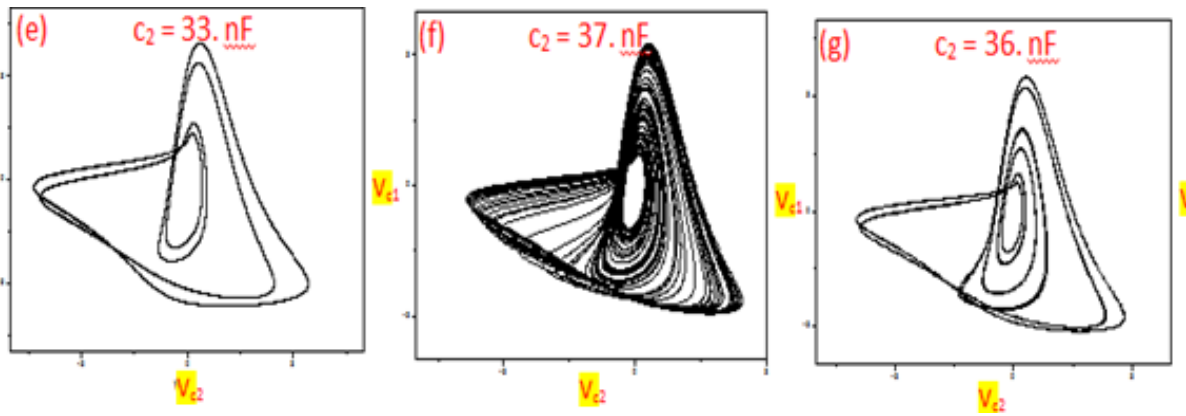

leads to an increase in the chaotic behavior by

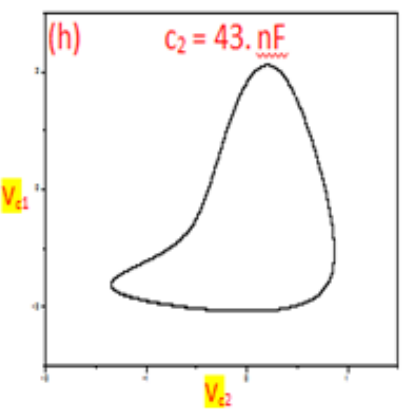

fixing the first and second capacities. As for the increase in the first

capacitance maps, as well as the bifurcation diagram and the phase portrait diagram of the circuit in the case of changing the value of the coil, we conclude that increasing the value of the coil with the proven value of the coil and the second of capacitance, we find that there is a decrease in chaotic behavior and the 
predominance of periodic behavior. We also note that increasing the second capacitance by confirming the value of the coil and the first capacitance leads to a decrease in chaotic behavior and also leads to the transition from dynamical behavior to stability.

\section{References}

[1] S. Rasappan, N. Kumar, S. Kumaravel, "control of colpitts-oscillator via adaptive feedback control, "Malaysian journal of mathematical sciences, vol.10, no.1, pp. 49-60, 2016.

[2] Z.-A. S. A Rahman, H. A. A. AlKashoash, S. M. Ramadhan, and Y. I. A. Al-Yasir, "Adaptive Control Synchronization of a Novel Memristive Chaotic System for Secure Communication Applications," Inventions, vol. 4, no. 2, p. 30, 2019.

[3] Y. Nishio and A. Ushida, "Quasisynchronization phenomena in chaotic circuits coupled by one resistor," IEEE Trans. Circuits Syst. I Fundam. Theory Appl., vol. 43, no. 6, pp. 491-496, 1996.

[4] Abo-Talib, A. (2020). Applying many of the methods to synchronization of chaos on the electronic circuit chaotic from type memductance. Al-Qadisiyah Journal of Pure Science, vol.26, no.1, pp. 1-13.
[5] C. Zhu, G. Wang , K. Sun, "Cryptanalysis and Improvement on an Image Encryption Algorithm Design Using a Novel Chaos Based S-Box, "Symmetry, vol.10, no.9, pp. 1-15, 2018.

[6] L. Chua, "Chua's circuit ten years later, "IEICE Trans. Fundamentals, vol. E77, no.11, pp 1811-1820, 1994.

[7] T. Matsumoto, "A chaotic attractor from Chua's circuit," IEEE Trans. Circuits Syst. vol. 31,no.12, pp.1055-1058, 1984.

[8] I. Pehlivan,Y. Glu, "A new chaotic attractor from general Lorenz system family and its electronic experimental implementation, "Turk. J. Electr. Eng. Comp. Sci, vol. 18, no. 2, pp. 171-184, 2010.

[9] O.E. Rossler, "An equation for continuous chaos, "Physics Letters A, vol. 57, no.5, pp. 397-398, 1976.

[10] K. Thamilmaran, M. Lakshmanan, and A. Venkatesan, "Hyperchaos in a modified canonical Chua's circuit," Int. J. Bifurc. Chaos, vol. 14, no. 01, pp. 221-243, 2004.

[11] F. Ahmed, "Non stability and chaos in Lasers ray Medium infrared, "thesis: submitted to the college of education university of basrah, 2001. 
[12] J. Abdullah, "Effect control parameter on behavior dynamics for laser succession It has three levels, "thesis: submitted to the college of education - university of basrah, 2007.

[13] C. Volos, I. Kyprianidis, I. Stouboulos, "Complex Dynamics Of a Memristor Based Chua's Canonical Circuit,"Proc. Of the $15^{\text {th }}$ WSEAS Int.conference on communication, $\mathrm{pp}$. 111-116, 2011.

[14] C. Volos, I. Kyprianidis, I. Stouboulos: Memristors, "A New Approach in Nonlinear Circuits Design, "Proc. Of the $14^{\text {th }}$ WSEAS Int. conference on communication, pp. 25-30, 2011.

[15] A.Y. Abbas, N.H. Noor, F. Ahmed, "Numerical Analysis of Resistance and conductance controlled the dynamical behavior of chaotic circuit, "American international journal research in science technology engineering and mathematics,vol.8,no.1, pp. 17-21, 2014.

[16] A.Y. Abbas, N.H. Noor, F. Ahmed, "Numerical Analysis of chaotic circuit under the influence of one component change, “international journal of American technology in computational and applied sciences, vol.10, no.1, pp. 5660,2014 . 